\title{
Solving second-order linear differential equations with random analytic coefficients about ordinary points: A full probabilistic solution by the first probability density function
}

\author{
J.-C. Cortés, A. Navarro-Quiles, J.-V. Romero*, M.-D. Roselló \\ Instituto Universitario de Matemática Multidisciplinar, Universitat Politècnica de València, Camino de Vera s/n, Valencia 46022, Spain
}

\section{A R T I C L E I N F O}

\section{Keywords:}

Random variable transformation technique

Second-order random linear differential

equation

Ordinary point

First probability density function

\begin{abstract}
A B S T R A C T
This paper deals with the approximate computation of the first probability density function of the solution stochastic process to second-order linear differential equations with random analytic coefficients about ordinary points under very general hypotheses. Our approach is based on considering approximations of the solution stochastic process via truncated power series solution obtained from the random regular power series method together with the so-called Random Variable Transformation technique. The validity of the proposed method is shown through several illustrative examples.
\end{abstract}

(c) 2018 Elsevier Inc. All rights reserved.

\section{Introduction and motivation}

The aim of this paper is to provide a full probabilistic description, through the approximation of the first probability density function (1-p.d.f.), $f_{1}(x, t)$, of the solution stochastic process (s.p.), $X(t)$, to the second-order random linear differential equation

$$
X^{\prime \prime}(t)+p(t ; A) X^{\prime}(t)+q(t ; A) X(t)=0, \quad t>t_{0} \in \mathbb{R},
$$

with initial conditions

$$
X\left(t_{0}\right)=Y_{0}, \quad X^{\prime}\left(t_{0}\right)=Y_{1} .
$$

In the initial value problem (IVP) (1) and (2), $A, Y_{0}$ and $Y_{1}$ are assumed to be absolutely continuous real random variables (r.v.'s) defined on a common complete probability space $(\Omega, \mathcal{F}, \mathbb{P})$. For the sake of clarity, realizations for any r.v., say $Z: \Omega \longrightarrow \mathcal{D}_{Z} \subset \mathbb{R}$, will be denoted by $z(\omega) \in \mathcal{D}_{Z}, \omega \in \Omega$. As usual, from now on $\mathcal{D}_{Z}$ will be referred to as the range or codomain of $Z$. To provide more generality in our study, we will assume that these r.v.'s are statistically dependent, being $f_{A, Y_{0}, Y_{1}}\left(a, y_{0}, y_{1}\right)$ the joint probability density function (p.d.f.) of the random vector $\left(A, Y_{0}, Y_{1}\right)$. The domain of this function will be denoted by $\mathcal{D}_{A, Y_{0}, Y_{1}} \subset \mathbb{R}^{3}$. For convenience, we also introduce the following notation, that will be used later, $\mathcal{D}_{A} \subset \mathbb{R}$, $\mathcal{D}_{Y_{1}} \subset \mathbb{R}$ and $\mathcal{D}_{A, Y_{1}} \subset \mathbb{R}^{2}$, that stand for the codomains of r.v.'s $A$ and $Y_{1}$, and random vector $\left(A, Y_{1}\right)$, respectively. Throughout

\footnotetext{
* Corresponding author.

E-mail addresses: jccortes@imm.upv.es (J.-C. Cortés), annaqui@doctor.upv.es (A. Navarro-Quiles), jvromero@imm.upv.es, jvromero@mat.upv.es (J.-V. Romero), drosello@imm.upv.es (M.-D. Roselló).
} 
this paper, we will assume that

H1 :

$f_{A, Y_{0}, Y_{1}}\left(a, y_{0}, y_{1}\right)$ is continuous in $y_{0}$ and bounded, i.e.,

$$
\exists M_{f}>0:\left|f_{A, Y_{0}, Y_{1}}\left(a, y_{0}, y_{1}\right)\right|<M_{f}, \forall\left(a_{0}, y_{0}, y_{1}\right) \in \mathcal{D}_{A, Y_{0}, Y_{1}} .
$$

It is remarkable to point out that a wide range of probabilistic distributions satisfy hypothesis H1, for example, gaussian, uniform, beta, gamma, log-normal, chi-square, t-student, etc. In particular, this condition is met for any multimodal distribution.

Henceforth, we will assume that coefficients $p(t ; A)$ and $q(t ; A)$ are s.p.'s, which depend on r.v. $A$, such that they are analytic about $\left(t_{0} ; a_{0}(\omega)\right)$ for every $a_{0}(\omega) \in \mathcal{D}_{A}, \omega \in \Omega$, i.e.,

H2 :

$$
\text { there exists a neighbourhood } \mathcal{N}_{p, q}\left(t_{0} ; a_{0}(\omega)\right) \subset\left[t_{0},+\infty\left[\times \mathcal{D}_{A}\right. \text { where }\right.
$$

$$
p(t ; A), q(t ; A) \text { are analytic } \forall a_{0}(\omega) \in \mathcal{D}_{A}, \omega \in \Omega \text {. }
$$

With the standard identification $p(t ; A) \equiv p(t ; a(\omega)), a(\omega) \in \mathcal{D}_{A}, \forall \omega \in \Omega$, we remember that the s.p. $p(t ; A)$ is analytic about $\left(t_{0}, a_{0}(\omega)\right), a_{0}(\omega) \in \mathcal{D}_{A}, \forall \omega \in \Omega$, if the deterministic function $p(t ; a(\omega))$ is analytic about $\left(t_{0}, a_{0}(\omega)\right)$. The same can be said for the s.p. $q(t ; A)$. To facilitate our subsequent analysis, hereinafter we will assume that $p(t ; A)$ and $q(t ; A)$ satisfy sufficient conditions in order to guarantee the IVP (1) and (2) has a unique solution.

Notice that in order to simplify notation, we have assumed that $p(t ; A)$ and $q(t ; A)$ are analytic in a common neighbourhood, $\mathcal{N}_{p, q}\left(t_{0} ; a_{0}(\omega)\right)$, which in practice will be defined by the intersection of the corresponding domains of analyticity of $p(t ; A)$ and $q(t ; A)$.

With the aim of motivating our study, below we point out two main facts. First, the interest of problem (1) and (2) that we are going to deal with and, secondly, the usefulness of computing the 1-p.d.f., $f_{1}(x, t)$. On the one hand, problems of the form (1) and (2) are important since many differential equations appearing in Mathematical Physics fall into this class. In this regard, it is worth pointing out that some recent contributions dealing with particular random differential equations of the form (1) and (2) can be found in [1-3], for example. In [1,2] authors give solutions of Hermite and Airy random differential equations, respectively, using the so-called mean square and mean fourth calculus [4,5]. In both contributions, approximations for the mean and the variance of the solution s.p. are given. In [3], these two statistical moments are computed for a wide range of second-order random linear differential equations taking advantage of homotopy analysis method. Recently, in [6] an analogous study has been conducted by applying the random differential transformation method developed in [7]. On the other hand, the computation of the 1-p.d.f. of the solution s.p. is advantageous since from it all one-dimensional statistical moments of the solution can be computed,

$$
\mathbb{E}\left[(X(t))^{k}\right]=\int_{-\infty}^{+\infty}(x(t))^{k} f_{1}(x, t) \mathrm{d} x, \quad k=1,2, \ldots
$$

Hence, the mean $\mu_{X}(t)=\mathbb{E}[X(t)]$ and the variance, $\sigma_{X}^{2}(t)=\mathbb{V}[X(t)]=\mathbb{E}\left[(X(t))^{2}\right]-\left(\mu_{X}(t)\right)^{2}$, are easily obtained as particular cases. In addition, $f_{1}(x, t)$ allows us to compute the probability that the solution lies in specific sets of interest,

$$
\mathbb{P}[\{\omega \in \Omega: a \leq X(t)(\omega) \leq b\}], \quad-\infty \leq a<b \leq+\infty .
$$

In this paper, the Random Variable Transformation (RVT) technique will be used to compute approximations of $f_{1}(x, t)$. For the sake of clarity in the presentation and notation, below we state a multidimensional version of RVT method.

Theorem 1 (Multidimensional Random Variable Transformation method). [5, p.25]. Let us consider $\mathbf{X}=\left(X_{1}, \ldots, X_{n}\right)^{\top}$ and $\mathbf{Z}=$ $\left(Z_{1}, \ldots, Z_{n}\right)^{\top}$ two $n$-dimensional absolutely continuous random vectors defined on a probability space $(\Omega, \mathfrak{F}, \mathbb{P})$. Let $\mathbf{r}: \mathbb{R}^{n} \rightarrow \mathbb{R}^{n}$ be a one-to-one deterministic transformation of $\mathbf{X}$ into $\mathbf{Z}$, i.e., $\mathbf{Z}=\mathbf{r}(\mathbf{X})$. Assume that $\mathbf{r}$ is continuous in $\mathbf{X}$ and has continuous partial derivatives with respect to each $X_{i}, 1 \leq i \leq n$. Then, if $f_{\mathbf{X}}(\mathbf{x})$ denotes the joint probability density function of random vector $\mathbf{X}$, and $\mathbf{s}=\mathbf{r}^{-1}=\left(s_{1}\left(z_{1}, \ldots, z_{n}\right), \ldots, s_{n}\left(z_{1}, \ldots, z_{n}\right)\right)^{\top}$ represents the inverse mapping of $\mathbf{r}=\left(r_{1}\left(x_{1}, \ldots, x_{n}\right), \ldots, r_{n}\left(x_{1}, \ldots, x_{n}\right)\right)^{\top}$, the joint probability density function of random vector $\mathbf{Z}$ is given by

$$
f_{\mathbf{Z}}(\mathbf{z})=f_{\mathbf{X}}(\mathbf{s}(\mathbf{z}))|J|,
$$

where $|J|$, which is assumed to be different from zero, is the absolute value of the Jacobian defined by the determinant

$$
J=\operatorname{det}\left(\frac{\partial \mathbf{s}^{\top}}{\partial \mathbf{z}}\right)=\operatorname{det}\left(\begin{array}{ccc}
\frac{\partial s_{1}\left(z_{1}, \ldots, z_{n}\right)}{\partial z_{1}} & \ldots & \frac{\partial s_{n}\left(z_{1}, \ldots, z_{n}\right)}{\partial z_{1}} \\
\vdots & \ddots & \vdots \\
\frac{\partial s_{1}\left(z_{1}, \ldots, z_{n}\right)}{\partial z_{n}} & \ldots & \frac{\partial s_{n}\left(z_{1}, \ldots, z_{n}\right)}{\partial z_{n}}
\end{array}\right) .
$$

In the context of solving random ordinary/partial differential and difference equations, RVT method has been successfully applied to compute, both analytically and numerically, the 1-p.d.f. associated to the solution (see for example, [8-20]). To the best of our knowledge, the application of RVT technique has not been explored yet in the context that approximations are obtained via power series. The aim of this paper is computing approximations of the 1-p.d.f., $f_{1}(x, t)$, of the solution s.p. 
to IVP (1) and (2), when the solution is constructed by power series using the so-called regular power series method [21, ch.4].

An important result that will be required later is the Poincaré's expansion theorem. For the sake of completeness, we remember its statement [22, Th. 9.2, p.119], [23, Th. 5.5, p.296]. This result permits to represent the solution of a differential equation whose formulation depends on a parameter, say $\lambda$, by means of a power series of the $\lambda$ parameter itself.

Theorem 2. Let us consider the initial value problem

$$
\left.\begin{array}{l}
\mathbf{W}^{\prime}(t)=\mathbf{f}(t, \mathbf{W}(t) ; \lambda), \quad t>t_{0}, \\
\mathbf{W}\left(t_{0}\right)=\mathbf{W}_{0}
\end{array}\right\}
$$

where $\mathbf{f}:] t_{0},+\infty\left[\times \mathbb{R}^{n} \times \mathbb{R} \longrightarrow \mathbb{R}^{n}, \mathbf{f}=\left[f_{1}, \ldots, f_{n}\right]^{\top}, \mathbf{W}(t) \equiv \mathbf{W}=\left[W_{1}, \ldots, W_{n}\right]^{\top}\right.$ and $\mathbf{W}_{0}=\left[W_{0,1}, \ldots, W_{0, n}\right]^{\top}$. If $\mathbf{f}(t, \mathbf{W} ; \lambda)$ admits a convergent power series expansion about $\left(t_{0}, \mathbf{W}_{0} ; \lambda_{0}\right)$, i.e.,

$$
f_{i}(t, \mathbf{W} ; \lambda)=\sum_{j \geq 0} \sum_{k_{1}, \ldots, k_{n} \geq 0} \sum_{l \geq 0} c_{j, k_{1}, \ldots, k_{n}, l}^{i}\left(t-t_{0}\right)^{j}\left(W_{1}-W_{0,1}\right)^{k_{1}} \ldots\left(W_{n}-W_{0, n}\right)^{k_{n}}\left(\lambda-\lambda_{0}\right)^{l}, \quad \lambda_{0} \in \mathbb{R}, \quad 1 \leq i \leq n,
$$

then, for every $\left(t_{0}, \mathbf{W}_{0}\right)$, the solution of the IVP (7) can also be represented as the following convergent power series

$$
\mathbf{W}(t)=\sum_{l \geq 0} \mathbf{L}_{l}(t)\left(\lambda-\lambda_{0}\right)^{l} .
$$

The coefficients $\mathbf{L}_{l}(t)$ are solutions of certain coupled unhomogeneous linear system of linear differential equations.

Remark 3. Notice that Theorem 2 can be applied to IVP (1) and (2) by considering the standard change of variable

$$
\mathbf{W}(t)=\left[W_{1}(t), W_{2}(t)\right]^{\top}=\left[X(t), X^{\prime}(t)\right]^{\top}, \quad \mathbf{W}_{0}=\left[Y_{0} Y_{1}\right]^{\top},
$$

$\lambda=a(\omega), \omega \in \Omega$ and $\mathbf{f}=\left[f_{1}(t, \mathbf{W}(t) ; a(\omega)), f_{2}(t, \mathbf{W}(t) ; a(\omega))\right]^{\top}$, being $f_{1}(t, \mathbf{W}(t) ; a(\omega))=W_{2}(t)$ and $f_{2}(t, \mathbf{W}(t) ; a(\omega))=$ $-p(t ; a(\omega)) W_{2}(t)-q(t ; a(\omega)) W_{1}(t)$, for each $\omega \in \Omega$.

We finish this section stating standard notation and some classical results related to uniform convergence that will be required later. Let $E \subset \mathbb{R}^{n}$ be a Lebesgue measurable set with finite measure. In the following, the pair $\left(\mathcal{L}^{1}(E),\|\cdot\|_{1}\right)$ will denote the normed space of mappings $f: E \subset \mathbb{R}^{n} \longrightarrow \mathbb{R}$ such that they are absolutely Lebesgue integrable, i.e.,

$$
\|f\|_{1}=\int_{E}|f|
$$

exists and is finite.

Proposition 1. [24, Prop.8, p.77] Let $E \subset \mathbb{R}^{n}$ be a Lebesgue measurable set with finite measure and let $\left\{f_{N}(e): N \geq 0\right\} \subset \mathcal{L}^{1}(E)$ such that $f_{N}(e)$ converges uniformly in $E$, i.e., $f_{N}(e) \underset{N \rightarrow+\infty}{\stackrel{\text { uniformly } E}{\longrightarrow}} f(e)$. Then, $f(e) \in \mathcal{L}^{1}(E)$ and

$$
\lim _{N \rightarrow+\infty} \int_{E} f_{N}(e) \mathrm{d} e=\int_{E} f(e) \mathrm{d} e .
$$

The following results are direct consequence of properties of uniform convergence, thus we omit their proofs.

Proposition 2. Let $\left\{g_{N}\left(z_{1}, z_{2}\right): N \geq 0\right\}$ and $\left\{h_{N}\left(z_{1}, z_{2}\right): N \geq 0\right\}$ be two sequences of real functions such that $h_{N}\left(z_{1}, z_{2}\right) \neq 0, \forall N \geq 0$, $\forall\left(z_{1}, z_{2}\right) \in D \subset \mathbb{R}^{2}$. Let us assume that both converge uniformly in $D$, i.e.,

$$
g_{N}\left(z_{1}, z_{2}\right) \underset{N \rightarrow+\infty}{\stackrel{\text { uniformly } D}{\longrightarrow}} g\left(z_{1}, z_{2}\right), \quad h_{N}\left(z_{1}, z_{2}\right) \stackrel{\text { uniformly } D}{\longrightarrow} h\left(z_{1}, z_{2}\right),
$$

being $h\left(z_{1}, z_{2}\right) \neq 0, \forall\left(z_{1}, z_{2}\right) \in D$, and there exist positive constants $M_{g}, m_{h}$ and $M_{h}$ such that

$$
\left|g_{N}\left(z_{1}, z_{2}\right)\right|<M_{g}, \quad 0<m_{h}<\left|h_{N}\left(z_{1}, z_{2}\right)\right|<M_{h}, \quad \forall N \geq 0, \forall\left(z_{1}, z_{2}\right) \in D .
$$

Then

$$
\frac{g_{N}\left(z_{1}, z_{2}\right)}{h_{N}\left(z_{1}, z_{2}\right)} \underset{N \rightarrow+\infty}{\stackrel{\text { uniformly } D}{\longrightarrow}} \frac{g\left(z_{1}, z_{2}\right)}{h\left(z_{1}, z_{2}\right)} .
$$

Proposition 3. Let $D_{u, v}$ be a subset of $\mathbb{R}^{2}$ such that

$$
\sup \left\{|v|:(u, v) \in D_{u, v}, \forall u\right\}<+\infty,
$$

and let $\left\{l_{N}(u): N \geq 0\right\}$ be a sequence of functions uniformly convergent in $D_{u}=\left\{u:(u, v) \in D_{u, v}\right\}$, i.e.,

$$
l_{N}(u) \underset{N \rightarrow+\infty}{\stackrel{\text { uniformly } D_{u}}{\longrightarrow}} l(u) .
$$


Then, the sequence of functions $b_{N}(u, v)=c+v l_{N}(u)$, where $c$ is a constant, converges uniformly in $D_{u, v}$, i.e.,

$$
b_{N}(u, v) \underset{N \rightarrow+\infty}{\stackrel{\text { uniformly } D_{u, v}}{\longrightarrow}} b(u, v),
$$

being $b(u, v)=c+v l(u)$.

Proposition 4. Let $\left\{\gamma_{N}\left(z_{1}, z_{2}\right): N \geq 0\right\}$ be a sequence of real functions such that converges uniformly in $D \subset \mathbb{R}^{2}$, i.e.,

$$
\gamma_{N}\left(z_{1}, z_{2}\right) \underset{N \rightarrow+\infty}{\stackrel{\text { uniformly } D}{\longrightarrow}} \gamma\left(z_{1}, z_{2}\right) \text {. }
$$

Let $\phi: D_{\phi} \subset \mathbb{R}^{3} \longrightarrow \mathbb{R}$ be a mapping such that is continuous with respect to its second variable. Then, the sequence of functions defined by $\psi_{N}\left(z_{1}, z_{2}\right)=\phi\left(z_{1}, \gamma_{N}\left(z_{1}, z_{2}\right), z_{2}\right)$ converges uniformly in $D$, i.e.,

$$
\psi_{N}\left(z_{1}, z_{2}\right) \underset{N \rightarrow+\infty}{\stackrel{\text { uniformly } D}{\longrightarrow}} \psi\left(z_{1}, z_{2}\right)
$$

where $\psi\left(z_{1}, z_{2}\right)=\phi\left(z_{1}, \gamma\left(z_{1}, z_{2}\right), z_{2}\right)$

This paper is organized as follows. In Section 2, an approximation of the first probability density function of the solution stochastic process to IVP (1) and (2) is constructed. This approximation is based on truncated random power series of the solution stochastic process obtained via regular power series method and the application of the Random Variable Transformation technique. Section 3 is addressed to show several illustrative examples where the proposed technique is successfully applied. Finally, in Section 4 our main conclusions are drawn.

\section{Computing the approximation of the first probability density function}

In agreement with regular power series method [21, ch.4], it is well-known that the solution of IVP (1)-(2) can be represented in the form

$$
X(t)=Y_{0} S_{1}(t ; A)+Y_{1} S_{2}(t ; A), \quad t \geq t_{0},
$$

being

$$
S_{1}(t ; A)=\sum_{n \geq 0} C_{n}(A)\left(t-t_{0}\right)^{n}, \quad S_{2}(t ; A)=\sum_{n \geq 0} D_{n}(A)\left(t-t_{0}\right)^{n} .
$$

The coefficients $C_{n}(A)$ and $D_{n}(A)$ are recursively determined from initial conditions $Y_{0}$ and $Y_{1}$ and a second-order linear difference equation. It is worth pointing out that $S_{1}(t ; A)$ and $S_{2}(t ; A)$ have no common powers $\left(t-t_{0}\right)^{n}$, hence the indexes $n \geq 0$ that appear in the two series defining $S_{1}(t ; A)$ and $S_{2}(t ; A)$ in (11) really lie in disjoint subsets of non-negative integers $\mathbb{N}=\{0,1,2, \ldots\}$. This issue has been omitted in (11) just to simplify notation. Also notice that, from initial condition $X\left(t_{0}\right)=$ $Y_{0}$ (see (2)) and (10) and (11), we can assure that $C_{0}(A)=1$ and $D_{0}(A)=0$.

Remark 4. On the one hand, interpreting the r.v. $A$ as a parameter indexed by $\omega \in \Omega, A=\{a(\omega): \omega \in \Omega\}$, and under hypothesis H2 given in (4), Theorem 2 guarantees that the solution $X(t)$ can also be represented as a power series of parameter $a(\omega), \omega \in \Omega$. Therefore, $S_{1}(t ; A)$ and $S_{2}(t ; A)$ do. On the other hand, taking into account the uniqueness of the solution of IVP (1) and (2), both series expansions (as powers of $t-t_{0}$ and as powers of $a(\omega)-a_{0}(\omega)$ ) match. Thus, the two series $S_{1}(t ; A)$ and $S_{2}(t ; A)$, given by (11), converge in certain domains $\mathcal{N}_{S_{1}}\left(t_{0} ; a_{0}(\omega)\right)$ and $\mathcal{N}_{S_{2}}\left(t_{0} ; a_{0}(\omega)\right)$, respectively, for all $a_{0}(\omega) \in \mathcal{D}_{A}, \omega \in \Omega$. In addition, uniform convergence takes place in every closed set contained in $\mathcal{N}_{S_{1}}\left(t_{0} ; a_{0}(\omega)\right)$ and $\mathcal{N}_{S_{2}}\left(t_{0} ; a_{0}(\omega)\right)$. Notice that the domain of convergence, $\mathcal{N}_{X}\left(t_{0} ; a_{0}(\omega)\right)$, of the power series solution $X(t)$ given in $(10)$, satisfies $\mathcal{N}_{p, q}\left(t_{0} ; a_{0}(\omega)\right) \subseteq \mathcal{N}_{X}\left(t_{0} ; a_{0}(\omega)\right)=\mathcal{N}_{S_{1}}\left(t_{0} ; a_{0}(\omega)\right) \cap \mathcal{N}_{S_{2}}\left(t_{0} ; a_{0}(\omega)\right)$ for all $a_{0}(\omega) \in \mathcal{D}_{A}, \omega \in \Omega$, where $\mathcal{N}_{p, q}\left(t_{0} ; a_{0}(\omega)\right)$ is defined in hypothesis H2 (see (4)).

The approximation of the 1-p.d.f., $f_{1}(x, t)$, to the s.p. $X(t)$ given by (10) will be computed from the truncation, say $X_{N}(t)$, of $X(t)$,

$$
X_{N}(t)=Y_{0} S_{1}^{N}(t ; A)+Y_{1} S_{2}^{N}(t ; A),
$$

where

$$
S_{1}^{N}(t ; A)=\sum_{n=0}^{N} C_{n}(A)\left(t-t_{0}\right)^{n}, \quad S_{2}^{N}(t ; A)=\sum_{n=0}^{N} D_{n}(A)\left(t-t_{0}\right)^{n},
$$

being $N$ a non-negative integer previously fixed. Truncation is required to keep burden computation feasible in dealing with the approximation of the 1-p.d.f.

Remark 5. On the one hand, we have previously shown that $C_{0}(A)=1$, hence from (13) one gets

$$
S_{1}^{N}\left(t_{0} ; A\right)=C_{0}(A)=1 \neq 0, \quad \forall N \geq 0 .
$$


From Remark 4, we know that $S_{1}(t ; A)$ can be represented through a power series in $t-t_{0}$ and $a(\omega)-a_{0}(\omega)$ for every $a_{0}(\omega), \omega \in \Omega$. Hence, $S_{1}^{N}(t ; A)$ is continuous at $\left(t_{0}, a_{0}(\omega)\right)$ for each $a_{0}(\omega), \omega \in \Omega$, since it is a polynomial in both variables $(t$, $a(\omega)$ ). This guarantees the existence of a neighbourhood, that without loss of generality can be taken as the one introduced in Remark $4, \mathcal{N}_{X}\left(t_{0} ; a_{0}(\omega)\right)$, and a positive constant, $m_{S_{1}}$, such that

$$
0<m_{S_{1}}<\left|S_{1}^{N}(t ; a(\omega))\right|, \quad \forall(t, a(\omega)) \in \mathcal{N}_{X}\left(t_{0} ; a_{0}(\omega)\right), \omega \in \Omega, \forall a_{0}(\omega) \in \mathcal{D}_{A}, \quad \forall N \geq 0 .
$$

On the other hand, in agreement with Remark 4 we know that $S_{i}(t ; a), i=1,2$, are analytic in a common neighbourhood $\mathcal{N}_{X}\left(t_{0} ; a_{0}(\omega)\right)$ and that both series $S_{i}(t ; a), i=1,2$, converge uniformly in every closed set $\mathcal{N}_{X}^{*}\left(t_{0} ; a_{0}(\omega)\right)$ contained in $\mathcal{N}_{X}\left(t_{0} ; a_{0}(\omega)\right)$ for all $a_{0}(\omega) \in \mathcal{D}_{A}, \omega \in \Omega$. This guarantees the existence of a positive constant $M_{S}$, such that

$$
\left|S_{i}^{N}(t ; a(\omega))\right|<M_{S}, \quad \forall(t, a(\omega)) \in \mathcal{N}_{X}^{*}\left(t_{0} ; a_{0}(\omega)\right) \subset \mathcal{N}_{X}\left(t_{0} ; a_{0}(\omega)\right), \omega \in \Omega, \forall a_{0}(\omega) \in \mathcal{D}_{A}, \forall N \geq 0, i=1,2 .
$$

Summarizing, from (15) and (16) the following bound for $S_{1}^{N}(t ; A)$ is obtained

$$
0<m_{S_{1}}<\left|S_{1}^{N}(t ; a(\omega))\right|<M_{S}, \quad \forall(t, a(\omega)) \in \mathcal{N}_{X}^{*}\left(t_{0} ; a_{0}(\omega)\right), \omega \in \Omega, \forall a_{0}(\omega) \in \mathcal{D}_{A}, \forall N \geq 0 .
$$

Let $t \geq t_{0}$ be fixed and so that it belongs to the neighbourhood $\mathcal{N}_{X}\left(t_{0} ; a_{0}(\omega)\right)$ of $t_{0}$ defined in Remark 5. Notice that this set does not depend on $a_{0}(\omega)$ since (15)-(17) hold for every $a_{0}(\omega) \in \mathcal{D}_{A}, \omega \in \Omega$. In the context of Theorem 1 with $n=3$ and $\mathbf{X}=\left(A, Y_{0}, Y_{1}\right)^{\top}$, let us consider the mapping $\mathbf{r}: \mathbb{R}^{3} \longrightarrow \mathbb{R}^{3}$ defined on a subset of $\mathcal{D}_{A, Y_{0}, Y_{1}}$ such that for $t \geq t_{0}$ fixed, $(t, a(\omega))$, $\omega \in \Omega$, belongs to the set $\mathcal{N}_{X}^{*}\left(t_{0} ; a_{0}(\omega)\right)$ introduced in Remark 5 , whose components are given by

$$
z_{1}=r_{1}\left(a, y_{0}, y_{1}\right)=a, \quad z_{2}=r_{2}\left(a, y_{0}, y_{1}\right)=y_{0} S_{1}^{N}(t ; a)+y_{1} S_{2}^{N}(t ; a), \quad z_{3}=r_{3}\left(a, y_{0}, y_{1}\right)=y_{1} .
$$

Thus, r.v. $Z_{2}=X_{N}$, i.e., $Z_{2}$ corresponds to the truncated series solution defined by (12). Notice that for convenience, we have omitted in the notation the dependence on variable $t$ since it has previously been fixed. The inverse of mapping $\mathbf{r}$, denoted by $\mathbf{s}: \mathbb{R}^{3} \longrightarrow \mathbb{R}^{3}$, is given by

$$
a=s_{1}\left(z_{1}, z_{2}, z_{3}\right)=z_{1}, \quad y_{0}=s_{2}\left(z_{1}, z_{2}, z_{3}\right)=\frac{z_{2}-z_{3} S_{2}^{N}\left(t ; z_{1}\right)}{S_{1}^{N}\left(t ; z_{1}\right)}, \quad y_{1}=s_{3}\left(z_{1}, z_{2}, z_{3}\right)=z_{3} .
$$

Taking into account (14), the absolute value of the Jacobian of this transformation, given by $|J|=\frac{1}{\left|S_{1}^{N}\left(t ; z_{1}\right)\right|}=\frac{1}{\left|S_{1}^{N}(t ; a)\right|} \neq 0$, is well-defined. Therefore, applying Theorem 1 , the joint p.d.f. of random vector $\left(Z_{1}, Z_{2}, Z_{3}\right)^{\top}$ is

$$
f_{Z_{1}, Z_{2}, Z_{3}}\left(z_{1}, z_{2}, z_{3}\right)=f_{A, Y_{0}, Y_{1}}\left(z_{1}, \frac{z_{2}-z_{3} S_{2}^{N}\left(t ; z_{1}\right)}{S_{1}^{N}\left(t ; z_{1}\right)}, z_{3}\right)\left|\frac{1}{S_{1}^{N}\left(t ; z_{1}\right)}\right|
$$

Then, marginalizing with respect to $Z_{1}=A$ and $Z_{3}=Y_{1}$, one obtains the p.d.f. of r.v. $Z_{2}=X_{N}$

$$
f_{Z_{2}}\left(z_{2}\right)=\iint_{\mathcal{D}_{A, Y_{1}}} f_{A, Y_{0}, Y_{1}}\left(a, \frac{z_{2}-y_{1} S_{2}^{N}(t ; a)}{S_{1}^{N}(t ; a)}, y_{1}\right)\left|\frac{1}{S_{1}^{N}(t ; a)}\right| \mathrm{d} y_{1} \mathrm{~d} a .
$$

As $t \geq t_{0}$ is arbitrary, this latter expression really gives the 1-p.d.f. of the truncated solution s.p. $X_{N}(t)$ to IVP (1) and (2)

$$
f_{1}^{N}(x, t)=\iint_{\mathcal{D}_{A, Y_{1}}} f_{A, Y_{0}, Y_{1}}\left(a, \frac{x-y_{1} S_{2}^{N}(t ; a)}{S_{1}^{N}(t ; a)}, y_{1}\right)\left|\frac{1}{S_{1}^{N}(t ; a)}\right| \mathrm{d} y_{1} \mathrm{~d} a .
$$

Below, we will provide conditions under which the approximation $f_{1}^{N}(x, t)$ converges to the exact 1-p.d.f., $f_{1}(x, t)$, i.e.,

$$
\lim _{N \rightarrow+\infty} f_{1}^{N}(x, t)=f_{1}(x, t), \quad \text { for each }(x, t) \in \mathbb{R} \times\left[t_{0},+\infty[\text { fixed, }\right.
$$

being

$$
f_{1}(x, t)=\iint_{\mathcal{D}_{A, Y_{1}}} f_{A, Y_{0}, Y_{1}}\left(a, \frac{x-y_{1} S_{2}(t ; a)}{S_{1}(t ; a)}, y_{1}\right)\left|\frac{1}{S_{1}(t ; a)}\right| \mathrm{d} y_{1} \mathrm{~d} a,
$$

where $S_{1}(t ; a)$ and $S_{2}(t ; a)$ are defined in (11).

Notice that, we can formally write

$$
\begin{aligned}
\lim _{N \rightarrow+\infty} f_{1}^{N}(x, t) & =\lim _{N \rightarrow+\infty} \iint_{\mathcal{D}_{A, Y_{1}}} f_{A, Y_{0}, Y_{1}}\left(a, \frac{x-y_{1} S_{2}^{N}(t ; a)}{S_{1}^{N}(t ; a)}, y_{1}\right)\left|\frac{1}{S_{1}^{N}(t ; a)}\right| \mathrm{d} y_{1} \mathrm{~d} a \\
& \stackrel{(\mathrm{I})}{=} \iint_{\mathcal{D}_{A, Y_{1}}} \lim _{N \rightarrow+\infty}\left(f_{A, Y_{0}, Y_{1}}\left(a, \frac{x-y_{1} S_{2}^{N}(t ; a)}{S_{1}^{N}(t ; a)}, y_{1}\right)\left|\frac{1}{S_{1}^{N}(t ; a)}\right|\right) \mathrm{d} y_{1} \mathrm{~d} a \\
& \stackrel{(\mathrm{II})}{=} \iint_{\mathcal{D}_{A, Y_{1}}} f_{A, Y_{0}, Y_{1}}\left(a, \lim _{N \rightarrow+\infty} \frac{x-y_{1} S_{2}^{N}(t ; a)}{S_{1}^{N}(t ; a)}, y_{1}\right)\left|\frac{1}{\lim _{N \rightarrow+\infty} S_{1}^{N}(t ; a)}\right| \mathrm{d} y_{1} \mathrm{~d} a
\end{aligned}
$$




$$
\begin{aligned}
& =\iint_{\mathcal{D}_{A, Y_{1}}} f_{A, Y_{0}, Y_{1}}\left(a, \frac{x-y_{1} S_{2}(t ; a)}{S_{1}(t ; a)}, y_{1}\right)\left|\frac{1}{S_{1}(t ; a)}\right| \mathrm{d} y_{1} \mathrm{~d} a \\
& =f_{1}(x, t) .
\end{aligned}
$$

As a result, we must legitimate steps (I) and (II) in (21) to justify (19). This will be done by assuming that conditions shown in Remark 5 are fulfilled for $t \geq t_{0}$ and $x \in \mathbb{R}$. We shall first justify the commutation between the limit as $N \rightarrow+\infty$ and the joint p.d.f. $f_{A, Y_{0}, Y_{1}}$ (step (II) in (21)), and secondly, the commutation between the limit as $N \rightarrow+\infty$ and the double integral (step (I) in (21)).

Firstly, observe that step (II) in (21) is valid because of the continuity of the joint p.d.f. $f_{A, Y_{0}, Y_{1}}\left(a, y_{0}, y_{1}\right)$ with respect to its second variable (see hypothesis $\mathrm{H} 1$ (see (3))).

Let us legitimate step (I) in (21). With this goal, we will first apply Proposition 1. This leads us to assume the following hypothesis hereinafter

H3 :

$$
\mathcal{D}_{A, Y_{1}} \text { is a Lebesgue measurable set of } \mathbb{R}^{2} \text { with finite measure }
$$

$$
\text { such that } \hat{M}=\sup \left\{\left|y_{1}(\omega)\right|:\left(a(\omega), y_{1}(\omega)\right) \in \mathcal{D}_{A, Y_{1}}, \omega \in \Omega\right\}<+\infty .
$$

Let us denote

$$
k_{N}\left(a, y_{1}\right)=f_{A, Y_{0}, Y_{1}}\left(a, \frac{x-y_{1} S_{2}^{N}(t ; a)}{S_{1}^{N}(t ; a)}, y_{1}\right)\left|\frac{1}{S_{1}^{N}(t ; a)}\right|, \quad\left(a, y_{1}\right) \in \mathcal{D}_{A, Y_{1}},
$$

the integrand of (18). Then, taking into account Remark 5, and particularly the lower bound that appears in (17), one gets

$$
k_{N}\left(a, y_{1}\right) \leq \frac{1}{m_{S_{1}}} f_{A, Y_{0}, Y_{1}}\left(a, \frac{x-y_{1} S_{2}^{N}(t ; a)}{S_{1}^{N}(t ; a)}, y_{1}\right), \quad \forall\left(a, y_{1}\right) \in \mathcal{D}_{A, Y_{1}} .
$$

Hence, since $f_{A, Y_{0}, Y_{1}}\left(a, y_{0}, y_{1}\right)$ is a p.d.f., $k_{N}\left(a, y_{1}\right)$ defined by (23) is Lebesgue absolutely integrable in $\mathcal{D}_{A, Y_{1}}$, i.e, $k_{N}\left(a, y_{1}\right) \in$ $\mathcal{L}^{1}\left(\mathcal{D}_{A, Y_{1}}\right)$ (notice that $k_{N}\left(a, y_{1}\right)$ is non-negative all over its domain).

Now, we shall show that

$$
k_{N}\left(a, y_{1}\right) \stackrel{\text { uniformly } \mathcal{D}_{A, Y_{1}}}{\underset{N \rightarrow \infty}{\longrightarrow}} k\left(a, y_{1}\right)
$$

with

$$
k\left(a, y_{1}\right)=f_{A, Y_{0}, Y_{1}}\left(a, \frac{x-y_{1} S_{2}(t ; a)}{S_{1}(t ; a)}, y_{1}\right)\left|\frac{1}{S_{1}(t ; a)}\right| .
$$

With this goal, let us consider the following inequality, obtained by adding and subtracting the term $f_{A, Y_{0}, Y_{1}}\left(a, \frac{x-y_{1} S_{2}^{N}(t ; a)}{S_{1}^{N}(t ; a)}, y_{1}\right)\left|\frac{1}{S_{1}(t ; a)}\right|$

$$
\begin{aligned}
& \left|k_{N}\left(a, y_{1}\right)-k\left(a, y_{1}\right)\right|=\left|f_{A, Y_{0}, Y_{1}}\left(a, \frac{x-y_{1} S_{2}^{N}(t ; a)}{S_{1}^{N}(t ; a)}, y_{1}\right)\right| \frac{1}{S_{1}^{N}(t ; a)} \mid \\
& -f_{A, Y_{0}, Y_{1}}\left(a, \frac{x-y_{1} S_{2}(t ; a)}{S_{1}(t ; a)}, y_{1}\right)\left|\frac{1}{S_{1}(t ; a)}\right| \mid \\
& \leq\left|f_{A, Y_{0}, Y_{1}}\left(a, \frac{x-y_{1} S_{2}^{N}(t ; a)}{S_{1}^{N}(t ; a)}, y_{1}\right)\right|\left|\frac{1}{\left|S_{1}^{N}(t ; a)\right|}-\frac{1}{\left|S_{1}(t ; a)\right|}\right| \\
& +\frac{1}{\left|S_{1}(t ; a)\right|}\left|f_{A, Y_{0}, Y_{1}}\left(a, \frac{x-y_{1} S_{2}^{N}(t ; a)}{S_{1}^{N}(t ; a)}, y_{1}\right)-f_{A, Y_{0}, Y_{1}}\left(a, \frac{x-y_{1} S_{2}(t ; a)}{S_{1}(t ; a)}, y_{1}\right)\right| \text {. }
\end{aligned}
$$

Let $t \geq t_{0}$ and $x \in \mathbb{R}$ be fixed, $N \geq 0$ an arbitrary integer, and consider $(t, a) \equiv(t, a(\omega)) \in \mathcal{N}_{X}^{*}\left(t_{0} ; a_{0}(\omega)\right), \omega \in \Omega$, for all $a_{0}(\omega) \in$ $\mathcal{D}_{A}$. Observe that the set $\mathcal{N}_{X}^{*}\left(t_{0} ; a_{0}(\omega)\right)$ has been previously introduced in Remark 5 . Let us make the following identification in Proposition 2: $z_{1}=a, z_{2}$ arbitrary, $D=\left\{\left(a, z_{2}\right) \in \mathbb{R}^{2}: z_{2}\right.$ is arbitrary $\}, g_{N}\left(a, z_{2}\right)=g\left(a, z_{2}\right)=1, h_{N}\left(a, z_{2}\right)=S_{1}^{N}(t ; a)$, $h\left(a, z_{2}\right)=S_{1}(t ; a)$ (remember that $t \geq t_{0}$ is fixed and that by Remark $5, h_{N}\left(a, z_{2}\right) \times h\left(a, z_{2}\right) \neq 0$ for all $\left.\left(a, z_{2}\right) \in D\right) \forall N \geq 0$ and $\forall\left(a, z_{2}\right) \in D, M_{g}=1, m_{h}=m_{S_{1}}, M_{h}=M_{S}$ (see (17)). Taking into account that, obviously $g_{N}\left(a, z_{2}\right)$ converges uniformly to $g(a$, $z_{2}$ ) on $D, h_{N}\left(a, z_{2}\right)$ converges uniformly to $h\left(a, z_{2}\right)$ on $D$ (see Remark 5) and hypothesis H1 (see (3)), then for every $\epsilon>0$, there exists $N_{0}$, which depends on $\epsilon$, such that

$$
\left|f_{A, Y_{0}, Y_{1}}\left(a, \frac{x-y_{1} S_{2}^{N}(t ; a)}{S_{1}^{N}(t ; a)}, y_{1}\right)\right| \times\left|\frac{1}{\left|S_{1}^{N}(t ; a)\right|}-\frac{1}{\left|S_{1}(t ; a)\right|}\right| \leq M_{f} \times \frac{\epsilon}{2 M_{f}}=\frac{\epsilon}{2}, \quad \forall N \geq N_{0},
$$

independently of the values of $\left(a, y_{1}\right)$. 
In order to obtain an analogous result for the second term of the right-hand side of inequality (25), let us first apply Proposition 3 considering the following identification: $u=a, v=-y_{1}, c=x$ (remember that $x$ is fixed), $l_{N}(a)=S_{2}^{N}(t ; a)$ with $t \geq t_{0}$ fixed and such that $(t, a) \in \mathcal{N}_{X}^{*}\left(t_{0} ; a_{0}(\omega)\right), \omega \in \Omega$, being this set defined in Remark $5, l(a)=S_{2}(t ; a), b_{N}\left(a, y_{1}\right)=$ $x-y_{1} S_{2}^{N}(t ; a)$ and $b\left(a, y_{1}\right)=x-y_{1} S_{2}(t ; a)$. Taking into account that $l_{N}(a)$ converges uniformly to $l(a)$ on $\mathcal{N}_{X}^{*}\left(t_{0} ; a_{0}(\omega)\right)$ (see Remark 5) and hypothesis H3 (see (22)), one follows

$$
x-y_{1} S_{2}^{N}(t, a) \stackrel{\text { uniformly } \mathcal{D}_{A, Y_{1}}}{\underset{N \rightarrow \infty}{\longrightarrow}} x-y_{1} S_{2}(t, a), \quad(x, t) \in \mathbb{R} \times\left[t_{0},+\infty[\text { fixed, }\right.
$$

for all $\left(a, y_{1}\right) \in \mathcal{D}_{A, Y_{1}}$ such that $(t, a(\omega)) \in \mathcal{N}_{X}^{*}\left(t_{0} ; a_{0}(\omega)\right)$, for every $a_{0}(\omega) \in \mathcal{D}_{A}, \omega \in \Omega$. Now, we apply Proposition 2 taking $z_{1}=a, z_{2}=y_{1}, g_{N}\left(a, y_{1}\right)=x-y_{1} S_{2}^{N}(t, a), g\left(a, y_{1}\right)=x-y_{1} S_{2}(t ; a)$ (observe that $g_{N}\left(a, y_{1}\right)$ converges uniformly to $g\left(a, y_{1}\right)$ by (27)), $h_{N}\left(a, y_{1}\right)=S_{1}^{N}(t ; a), h\left(a, y_{1}\right)=S_{1}(t ; a)$ (by Remark 5 it is known that $h_{N}\left(a, y_{1}\right)$ converges uniformly to $h\left(a, y_{1}\right)$ and both are nonull), $M_{g}=|x|+\hat{M} \times M_{S}$ (see hypothesis H3 in (22)) and $M_{h}=M_{S}$ (see (16)), this justifies that

$$
\frac{x-y_{1} S_{2}^{N}(t, a)}{S_{1}^{N}(t, a)} \underset{N \rightarrow \infty}{\stackrel{\text { uniformly } \mathcal{D}_{A, Y_{1}}}{\longrightarrow}} \frac{x-y_{1} S_{2}(t, a)}{S_{1}(t, a)}, \quad(x, t) \in \mathbb{R} \times\left[t_{0},+\infty[\text { fixed } .\right.
$$

Now, let us apply Proposition 4 with the following identification: $z_{1}=a, z_{2}=y_{1}, D \subset \mathbb{R}^{2}$ the set of $\left(a, y_{1}\right) \in \mathcal{D}_{A, Y_{1}}$ such that $\left(t, a(\omega) \in \mathcal{N}_{X}^{*}\left(t_{0} ; a(\omega)\right)\right.$ for every $a_{0}(\omega) \in \mathcal{D}_{A}, \omega \in \Omega$,

$$
\gamma_{N}\left(a, y_{1}\right)=\frac{x-y_{1} S_{2}^{N}(t ; a)}{S_{1}^{N}(t ; a)}, \quad \gamma\left(a, y_{1}\right)=\frac{x-y_{1} S_{2}(t ; a)}{S_{1}(t ; a)},
$$

$\phi \equiv f_{A, Y_{0}, Y_{1}}$ and

$$
\psi_{N}\left(a, y_{1}\right)=f_{A, Y_{0}, Y_{1}}\left(a, \frac{x-y_{1} S_{2}^{N}(t ; a)}{S_{1}^{N}(t ; a)}, y_{1}\right), \quad \psi\left(a, y_{1}\right)=f_{A, Y_{0}, Y_{1}}\left(a, \frac{x-y_{1} S_{2}(t ; a)}{S_{1}(t ; a)}, y_{1}\right) .
$$

Notice that, by (28), $\gamma_{N}\left(a, y_{1}\right)$ converges uniformly to $\gamma\left(a, y_{1}\right)$ in $D$, and by hypothesis $\mathrm{H} 1$ (see (3)), $\phi$ is a continuous mapping with respect to its second variable $y_{0}$. Then applying Proposition 4 one can assure that for every $\epsilon>0$, there exists $N_{0}$, which depends on $\epsilon$, such that

$$
\frac{1}{\left|S_{1}(t ; a)\right|}\left|f_{A, Y_{0}, Y_{1}}\left(a, \frac{x-y_{1} S_{2}^{N}(t ; a)}{S_{1}^{N}(t ; a)}, y_{1}\right)-f_{A, Y_{0}, Y_{1}}\left(a, \frac{x-y_{1} S_{2}(t ; a)}{S_{1}(t ; a)}, y_{1}\right)\right|<\frac{1}{m_{S_{1}}} \times \frac{m_{S_{1}} \epsilon}{2}=\frac{\epsilon}{2}, \quad \forall N \geq N_{0},
$$

independently of the values of $\left(a, y_{1}\right)$. Observe that in (29) we have used that from (17) one gets $0<m_{S_{1}} \leq\left|S_{1}(t ; a)\right|$ in the set where we are working. Therefore, from (25), (26) and (29), one deduces that for every $\epsilon>0$ there exists $N_{0}$, which depends on $\epsilon$ such that

$$
\left|k_{N}\left(a, y_{1}\right)-k\left(a, y_{1}\right)\right|<\epsilon, \quad \forall N \geq N_{0},
$$

independently of values of $\left(a, y_{1}\right)$. Hence, (24) is proved.

Summarizing, the following result has been established:

Theorem 6. Let us consider the IVP (1) and (2) and assume that

(i) The joint probability density function $f_{A, Y_{0}, Y_{1}}\left(a, y_{0}, y_{1}\right)$ of random input vector $\left(A, Y_{0}, Y_{1}\right)$ satisfies hypothesis $H 1$ (see (3)).

(ii) The coefficients $p(t ; A)$ and $q(t ; A)$ are stochastic processes satisfying hypothesis $H 2$ (see (4)).

(iii) The codomain of random vector $\left(A, Y_{1}\right), \mathcal{D}_{A, Y_{1}}$, verifies hypothesis $H 3$ (see (22)).

Let $S_{1}^{N}(t, a)$ and $S_{2}^{N}(t ; a)$ be truncated random power series solutions of the form (11) to the IVP (1) and (2). Then, $f_{1}^{N}(x, t)$ defined by (18) is the first probability density function of the approximation (12) of the solution stochastic process $X(t)$ given by (10) to the IVP (1) and (2). Furthermore, for each $(x, t), f_{1}^{N}(x, t)$ converges to the first probability density function $f_{1}(x, t)$ of $X(t)$.

\section{Examples}

This section is addressed to show two examples where the previous theoretical results are illustrated. In order to show the capability of our approach to deal with a variety of statistical distributions, in the first example we consider that input parameters have a joint p.d.f., whereas in the second example input parameters are assumed to be independent r.v.'s with different probability distributions.

Example 1. Let us consider the IVP

$$
\left.\begin{array}{l}
X^{\prime \prime}(t)+A X(t)=0, \quad t>0, \\
X(0)=Y_{0}, \\
X^{\prime}(0)=Y_{1},
\end{array}\right\}
$$



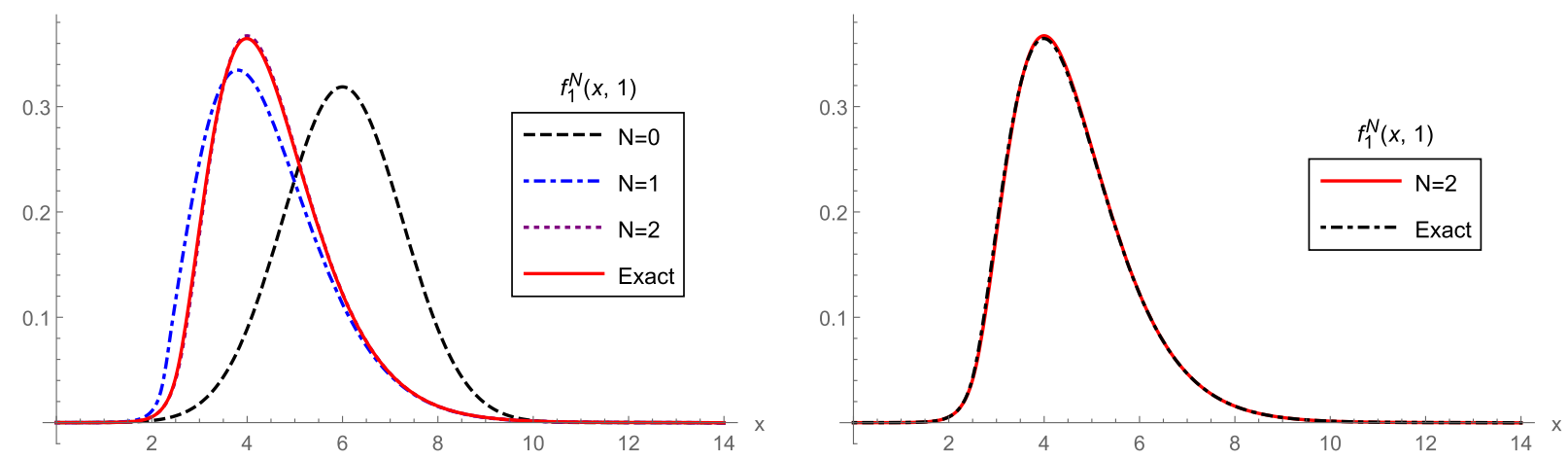

Fig. 1. Left: Comparison of the approximations, $f_{1}^{N}(x, t)$, of the 1-p.d.f. of the solution s.p. to the random IVP (1) taking as series order truncation $N \in\{0$, $1,2\}$ and the corresponding exact 1-p.d.f. $f_{1}(x, t)$ at $t=1$. Right: For ease of comparison, the function $f_{1}^{N}(x, 1)$, with $N=2$, has been plotted together with the exact 1-p.d.f. $f_{1}(x, 1)$.
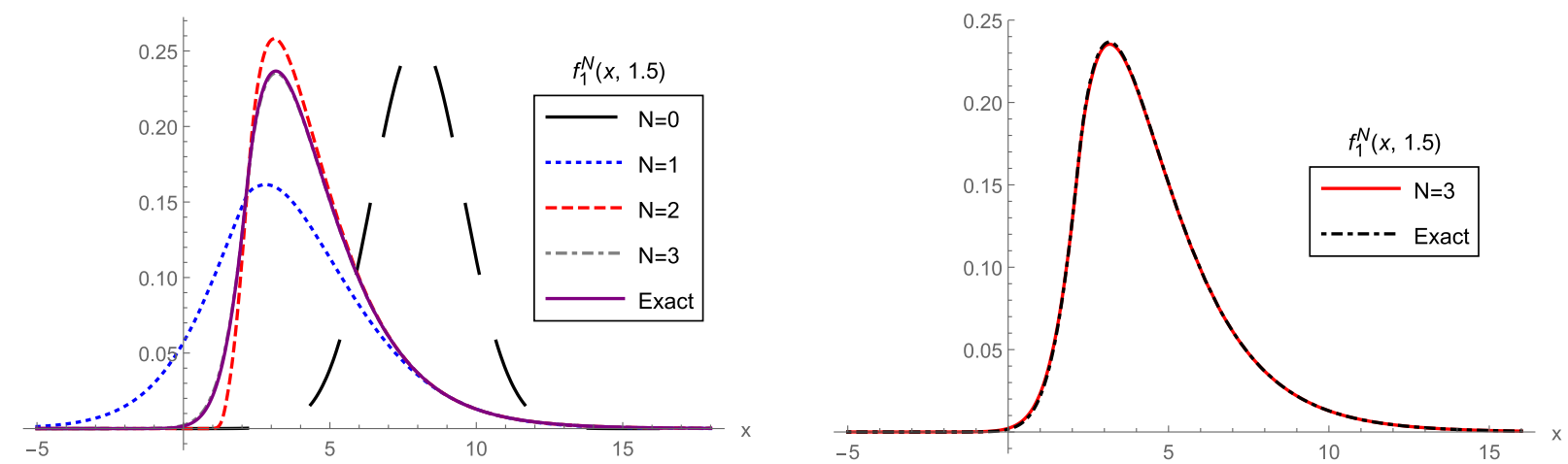

Fig. 2. Left: Comparison of the approximations, $f_{1}^{N}(x, t)$, of the 1-p.d.f. of the solution s.p. to the random IVP (1) taking as series order truncation $N \in\{0$, $1,2,3\}$ and the corresponding exact 1-p.d.f. $f_{1}(x, t)$ at $t=1.5$. Right: For ease of comparison, the function $f_{1}^{N}(x, 1.5)$, with $N=3$, has been plotted together with the exact 1-p.d.f. $f_{1}(x, 1.5)$.

where $\left(A, Y_{0}, Y_{1}\right)$ is a random vector with a multivariate normal distribution, $\left(A, Y_{0}, Y_{1}\right) \sim \mathrm{N}_{\mathrm{T}}(\boldsymbol{\mu} ; \boldsymbol{\Sigma})$, truncated on the domain $\mathrm{T}=[-2.4,4.4] \times[-3,9] \times[0.6,7.4] \subset \mathbb{R}^{3}$, i.e., $a(\omega) \in[-2.4,4.4], y_{0}(\omega) \in[-3,9]$ and $y_{1}(\omega) \in[0.6,7.4], \omega \in \Omega$, being $\boldsymbol{\mu}$ the mean vector and $\Sigma$ the variance-covariance matrix given by

$$
\boldsymbol{\mu}=(1,2,4)^{\top}, \quad \boldsymbol{\Sigma}=\left(\begin{array}{ccc}
1 / 2 & -1 / 4 & 1 / 3 \\
-1 / 4 & 2 / 3 & 1 / 5 \\
1 / 3 & 1 / 5 & 1 / 2
\end{array}\right) .
$$

By applying Theorem 1, it is straightforwardly to check that the exact 1 -p.d.f., $f_{1}(x, t)$, is given by

$$
f_{1}(x, t)=\int_{0.6}^{7.4} \int_{-2.4}^{4.4} f_{A, Y_{0}, Y_{1}}\left(a,\left(x-\frac{y_{1} \sin (\sqrt{a} t)}{\sqrt{a}}\right) \frac{1}{\cos (\sqrt{a} t)}, y_{1}\right) \frac{1}{|\cos (\sqrt{a} t)|} \mathrm{d} a \mathrm{~d} y_{1},
$$

where $f_{A, Y_{0}, Y_{1}}\left(a, y_{0}, y_{1}\right)$ denotes the joint p.d.f. of vector $\left(A, Y_{0}, Y_{1}\right) \sim \mathrm{N}_{\mathrm{T}}(\boldsymbol{\mu} ; \boldsymbol{\Sigma})$. In Figs. 1-3, we have plotted the exact 1-p.d.f., $f_{1}(x, t)$, and the approximations, $f_{1}^{N}(x, t)$, of the solution s.p. to IVP (1) for different values of the truncation order $N$ at the time instants $t \in\{1,1.5,2\}$, respectively. From these graphical representations, we observe that $f_{1}^{N}(x, t)$ tends to $f_{1}(x, t)$ as $N$ increases, for every value of $t$. Moreover, excellent approximations are achieved by considering a few terms. In this regard, for instance, in Fig. 1 one can observe that the approximation $f_{1}^{N}(x, t), N=2$, approximately coincides with the exact function $f_{1}(x, t)$ at $t=1$. The same can be said for the approximations $f_{1}^{N}(x, 1.5)$ and $f_{1}^{N}(x, 2)$, taking $N=3$ and $N=4$, (see Figs. 2 and 3, respectively). Naturally, the further away from the origin is $t$, the higher order $N$ is required to enhance the approximation. To facilitate the graphical assessment of approximations, the function $f_{1}^{N}(x, t)$, for the corresponding greatest value of $N$ and the corresponding $t$, has been plotted together with the exact 1-p.d.f., $f_{1}(x, t)$, in the right-hand side of Figs. $1-3$. In all cases we can see that $f_{1}^{N}(x, t)$ matches the exact 1-p.d.f. $f_{1}(x, t)$.

From Figs. $1-3$ it is observed that differences between the approximations $f_{1}^{N}(x, t)$ and the exact p.d.f. $f_{1}(x, t)$ become smaller as truncation order $N$ increases. To assess the quality of approximations, in Table 1 we show the figures for the following error measure

$$
e_{N}=\int_{-\infty}^{+\infty}\left|f_{1}^{N}(x, t)-f_{1}(x, t)\right| \mathrm{d} x, \quad N \geq 0, t \geq 0 \text { fixed },
$$



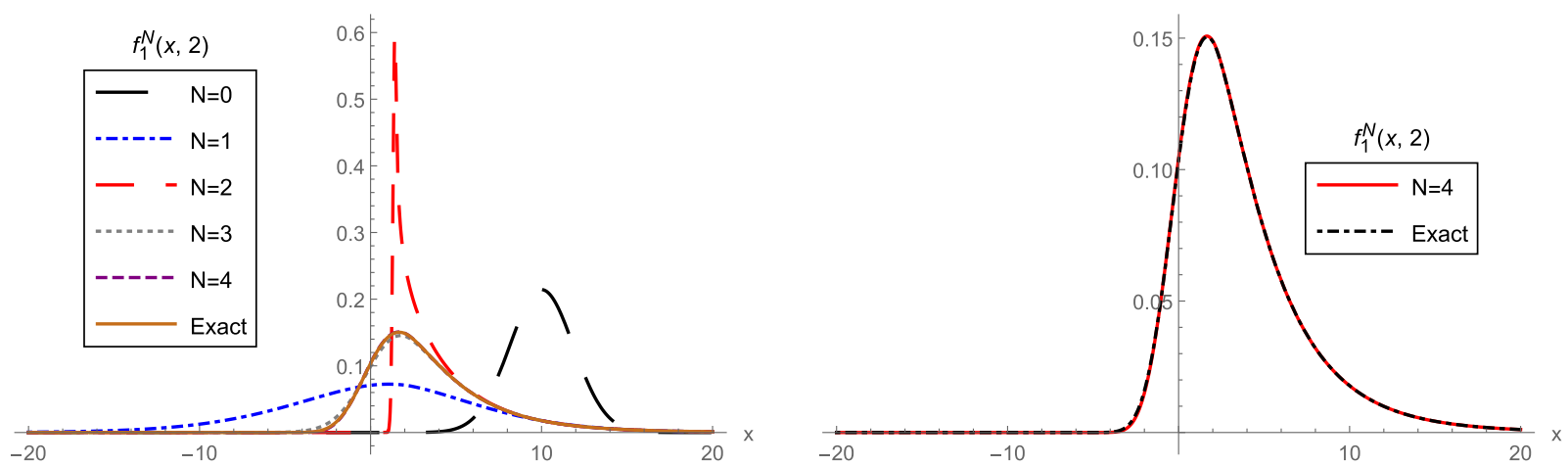

Fig. 3. Left: Comparison of the approximations, $f_{1}^{N}(x, t)$, of the 1-p.d.f. of the solution s.p. to the random IVP (1) taking as series order truncation $N \in\{0$ $1,2,3,4\}$ and the corresponding exact 1-p.d.f. $f_{1}(x, t)$ at $t=2$. Right: For ease of comparison, the function $f_{1}^{N}(x, 2)$, with $N=4$, has been plotted together with the exact 1-p.d.f. $f_{1}(x, 2)$.

Table 1

Error measure $e_{N}$ defined by (32) for different time instants, $t \in\{1,1.5,2\}$, and series truncation orders, $N \in\{0,1,2,3,4\}$ in Example 1 . For every $t$, we have taken $10^{-2}$ as stopping criterion for the tolerance error to $e_{N}$, then we have just collected values up to the error is for the first time less than $10^{-2}$. Otherwise, the error is still less than $10^{-2}$ and it has been denoted by means of an hyphen-.

\begin{tabular}{llllll}
\hline$e_{N}$ & $N=0$ & $N=1$ & $N=2$ & $N=3$ & $N=4$ \\
\hline$t=1.0$ & 0.963814 & 0.144837 & 0.00868924 & - & - \\
$t=1.5$ & 1.42046 & 0.418365 & 0.0835404 & 0.0055119 & - \\
$t=2.0$ & 1.6199 & 0.672689 & 0.539773 & 0.0356643 & 0.00364729 \\
\hline
\end{tabular}
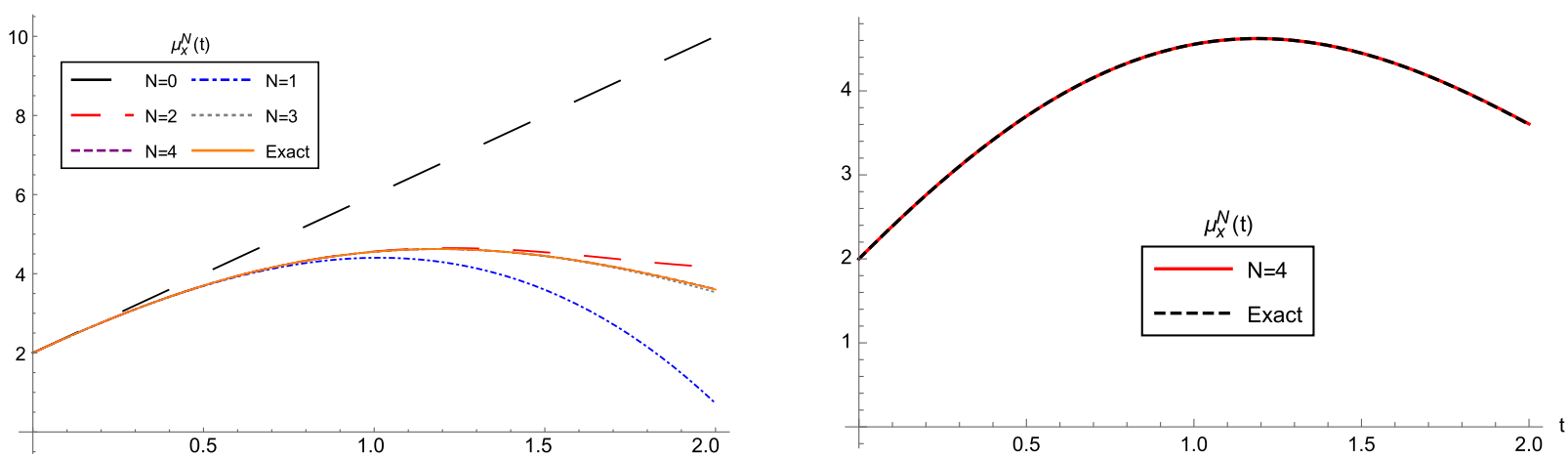

Fig. 4. Left: Comparison of exact mean, $\mu_{x}(t)$, and different approximations, $\mu_{x}^{N}(t)$, of the solution s.p. to the random IVP (30) taking as series truncation orders $N \in\{0,1,2,3,4\}$. Right: To facilitate the graphical assessment, exact mean and approximation for $N=4$ have been magnified.

Table 2

Values of errors for the mean, $e_{N}^{\mu}$, and the standard deviation, $e_{N}^{\sigma}$, given by (33) for $N \in\{0,1,2,3,4\}$, in Example 1.

\begin{tabular}{llllll}
\hline Error & $N=0$ & $N=1$ & $N=2$ & $N=3$ & $N=4$ \\
\hline$e_{N}^{\mu}$ & 4.11879 & 1.10359 & 0.156213 & 0.0151474 & 0.00105146 \\
$e_{N}^{\sigma}$ & 0.800605 & 0.833386 & 0.136366 & 0.0159361 & 0.00133905 \\
\hline
\end{tabular}

taking as stopping criterion for the tolerance error $10^{-2}$, it is, we have just collected values up to the error, $e_{N}$, is for the first time less than $10^{-2}$.

Finally, in Figs. 4 and 5 we compare the exact mean, $\mu_{x}(t)$, and the exact standard deviation, $\sigma_{x}(t)$, in the interval $t \in[0,2]$ with the corresponding approximations, $\mu_{x}^{N}(t)$ and $\sigma_{x}^{N}(t)$, respectively, for different truncation orders, $N$. The errors of these approximations are shown in Table 2. The errors have been calculated using the following expressions

$$
e_{N}^{\mu}=\int_{0}^{2}\left|\mu_{x}(t)-\mu_{x}^{N}(t)\right| \mathrm{d} t, \quad e_{N}^{\sigma}=\int_{0}^{2}\left|\sigma_{x}(t)-\sigma_{x}^{N}(t)\right| \mathrm{d} t .
$$



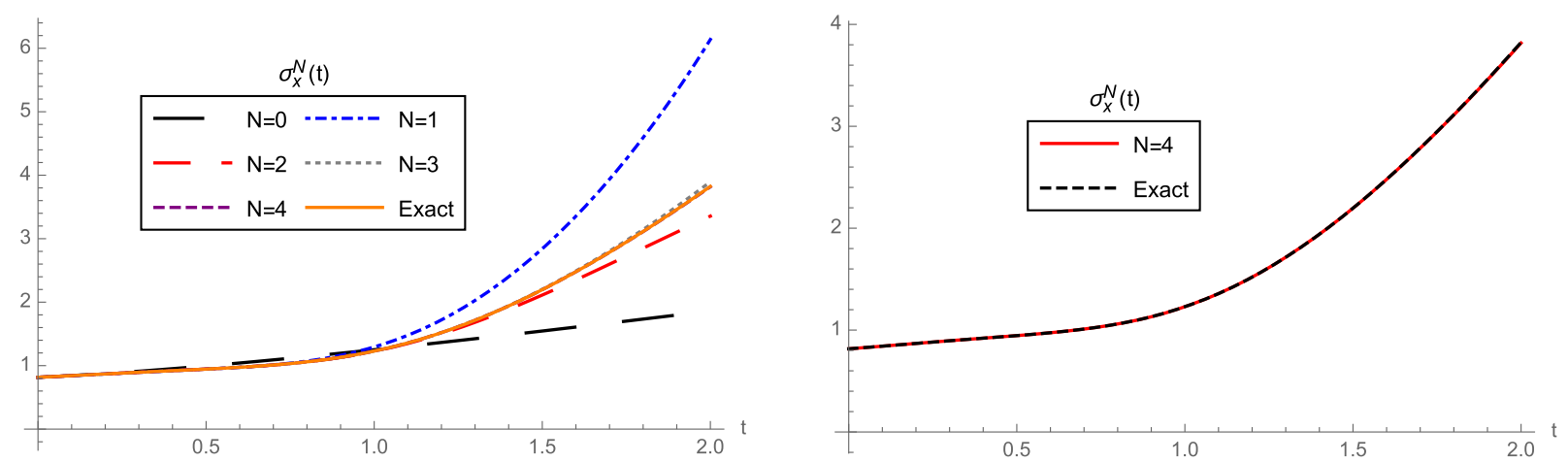

Fig. 5. Left: Comparison of exact standard deviation, $\sigma_{x}(t)$, and different approximations, $\sigma_{x}^{N}(t)$, of the solution s.p. to the random IVP (30) taking as series truncation orders $N \in\{0,1,2,3,4\}$. Right: To facilitate the graphical assessment, exact standard deviation and approximation for $N=4$ have been magnified.

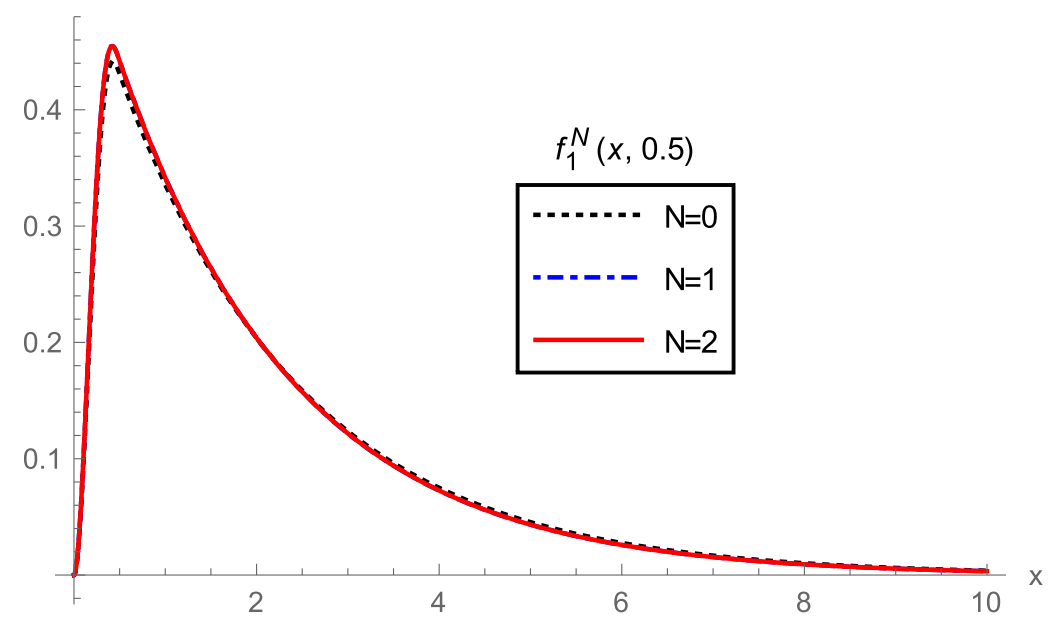

Fig. 6. Approximations, $f_{1}^{N}(x, 0.5)$, of the 1-p.d.f. of the solution s.p. to the random IVP (34) taking as series order truncation $N \in\{0,1,2\}$.

Example 2. Let us consider the Airy IVP

$$
\left.\begin{array}{l}
X^{\prime \prime}(t)+A t X(t)=0, \quad t>0, \\
X(0)=Y_{0}, \\
X^{\prime}(0)=Y_{1},
\end{array}\right\}
$$

where $A$ is a uniform r.v., $A \sim \mathrm{U}([1,2]), Y_{0}$ is an exponential r.v. of parameter $\lambda=1 / 2, Y_{0} \sim \operatorname{Exp}(1 / 2)$ and $Y_{1}$ is a beta r.v. of parameters $\alpha=2$ and $\beta=3, Y_{1} \sim \operatorname{Be}(2 ; 3)$. Hereinafter, $A, Y_{0}$ and $Y_{1}$ are assumed to be independent r.v.'s. To illustrate the capability of our approach to approximate the 1-p.d.f. of the solution s.p. to IVP (34), we have plotted the approximations, $f_{1}^{N}(x, t)$, for different values of $N$ and the time instants $t \in\{0.5,1.5 .2 .5\}$ (see Figs. 6,7 and 8 , respectively). In Fig. 6 , we can see that approximations corresponding to $N=3$ and $N=4$ match. To facilitate the graphical assessment of approximations, the functions $f_{1}^{N}(x, 1.5)$, with $N \in\{2,3,4\}$ and $f_{1}^{N}(x, 2.5)$, with $N \in\{4,5,6\}$ have been magnified on the right-hand side of Figs. 7 and 8 , respectively. From both graphical representations, we can see that the approximations $\left\{f_{1}^{3}(x, 1.5), f_{1}^{4}(x, 1.5)\right\}$ and $\left\{f_{1}^{5}(x, 2.5), f_{1}^{6}(x, 2.5)\right\}$ match, respectively.

From these graphical representations, we observe that differences between the approximations $f_{1}^{N+1}(x, t)$ and $f_{1}^{N}(x, t)$ are smaller as $N$ increases. As it also happens in the deterministic scenario, when the classical regular power series method is applied, we observe that very good approximations are achieved even for small values of the truncation order $N$. This assertion is numerically confirmed in Table 3 where the following error measure

$$
\hat{e}_{N}=\int_{-\infty}^{+\infty}\left|f_{1}^{N+1}(x, t)-f_{1}^{N}(x, t)\right| \mathrm{d} x, \quad N \geq 0, t \geq 0 \text { fixed }
$$

is reported. We have taken $10^{-2}$ as stopping criterion for the tolerance error to $\hat{e}_{N}$, then we have just collected values up to the error is for the first time less that $10^{-2}$.

In Figs. 9 and 10 we show the approximate mean, $\mu_{x}^{N}(t)$, and the approximate standard deviation, $\sigma_{x}^{N}(t)$, in the interval $t \in[0,2.75]$, for different orders of truncation, $N$, respectively. To account for the errors in Table 4 we use the following 

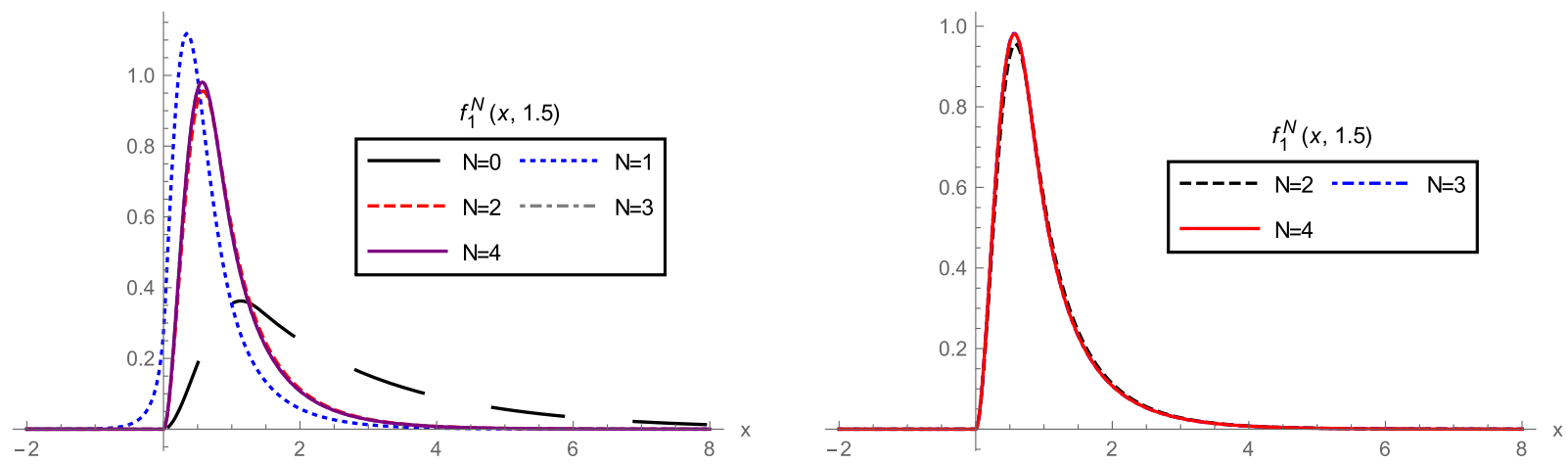

Fig. 7. Left: Approximations, $f_{1}^{N}(x, 1.5)$, of the 1 -p.d.f. of the solution s.p. to the random IVP (34) taking as series order truncation $N \in\{0,1,2,3,4\}$. Right: To facilitate the graphical assessment of approximations, the functions $f_{1}^{N}(x, 1.5)$, with $N \in\{2,3,4\}$, have been magnified.

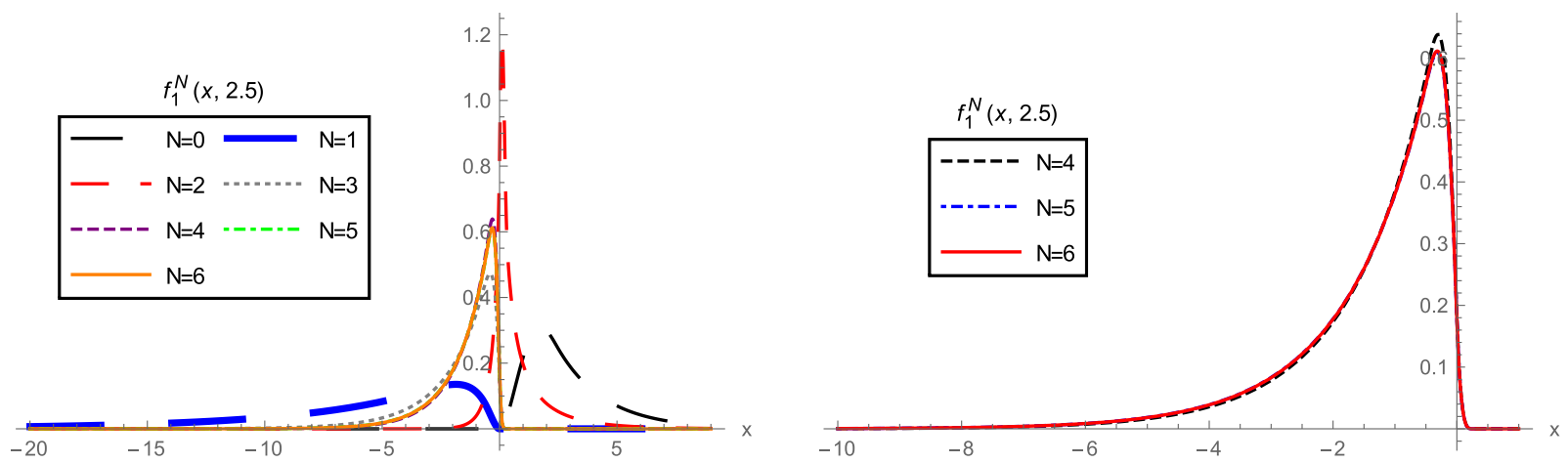

Fig. 8. Left: Approximations, $f_{1}^{N}(x, 2.5)$, of the 1 -p.d.f. of the solution s.p. to the random IVP $(34)$ taking as series order truncation $N \in\{0,1,2,3,4,5,6\}$. Right: To facilitate the graphical assessment of approximations, the functions $f_{1}^{N}(x, 2.5)$, with $N \in\{4,5,6\}$, have been magnified.

\section{Table 3}

Error measure $\hat{e}_{N}$ defined by (35) for different time instants, $t \in\{0.5,1.5,2.5\}$, and series truncation orders, $N \in\{0,1,2,3,4,5\}$ in Example 2. For every $t$, we have taken $10^{-2}$ as stopping criterion for the tolerance error to $\hat{e}_{N}$, then we have just collected values up to the error is for the first time less that $10^{-2}$. Otherwise, the error is still less than $10^{-2}$ and it has been denoted by means of an hyphen-.

\begin{tabular}{lllllll}
\hline$\hat{e}_{N}$ & $N=0$ & $N=1$ & $N=2$ & $N=3$ & $N=4$ & $N=5$ \\
\hline$t=0.5$ & 0.0245702 & 0.000159714 & - & - & - & - \\
$t=1.5$ & 1.25224 & 0.514115 & 0.0368461 & 0.00167046 & - & - \\
$t=2.5$ & 1.98864 & 1.86826 & 1.61559 & 0.227534 & 0.0338858 & 0.00285845
\end{tabular}
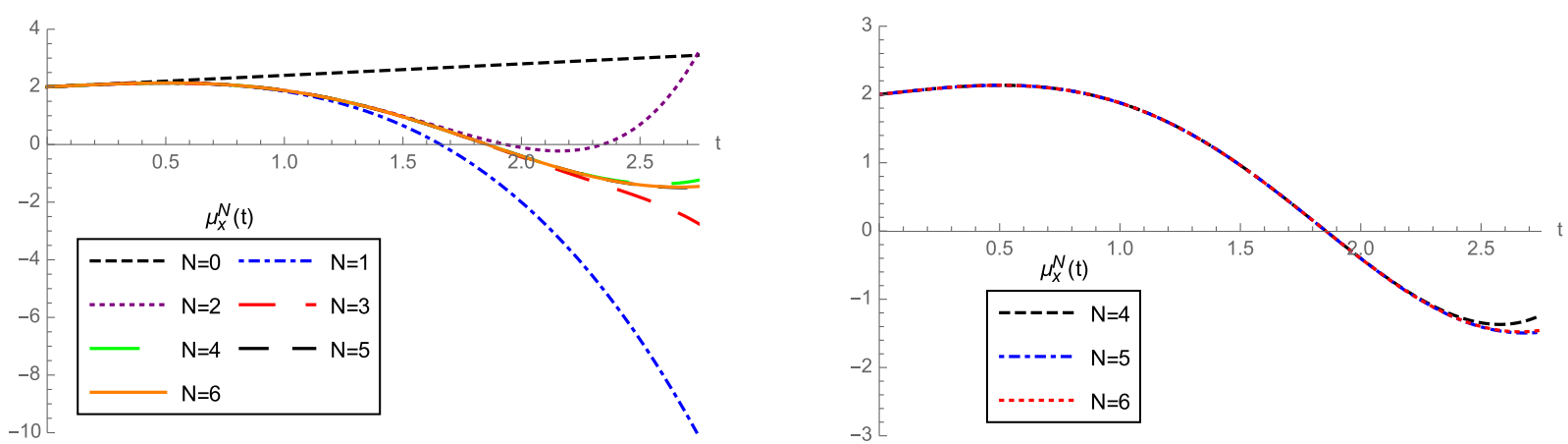

Fig. 9. Left: Approximations of the mean, $\mu_{x}^{N}(t)$, of the solution s.p. to the random IVP $(34)$ taking as series truncation orders $N \in\{0,1,2,3,4,5,6\}$. Right: To facilitate the graphical assessment, approximations to the mean, $\mu_{x}^{N}(t)$, with $N \in\{4,5,6\}$, have been magnified. 

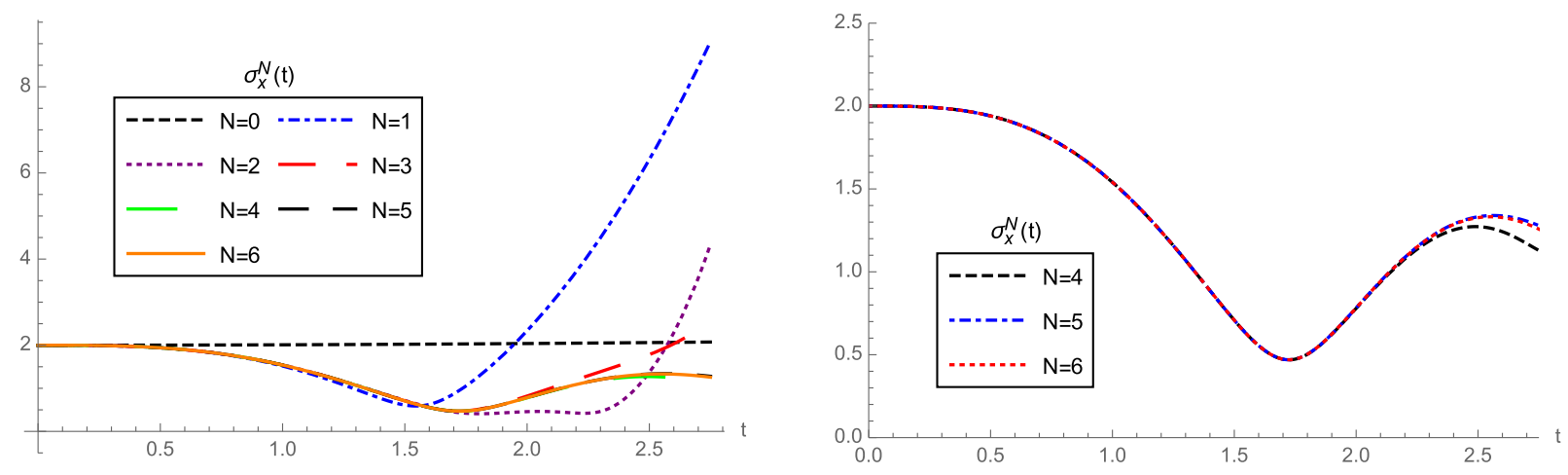

Fig. 10. Left: Approximations of the standard deviation, $\sigma_{x}^{N}(t)$, of the solution s.p. to the random IVP (34) taking as series truncation orders $N \in\{0,1,2,3$, $4,5,6\}$. Right: To facilitate the graphical assessment, approximations to the standard deviation, $\sigma_{x}^{N}(t)$, with $N \in\{4,5,6\}$, have been magnified.

Table 4

Values of errors for the approximate mean, $\hat{e}_{N}^{\mu}$, and the approximate standard deviation, $\hat{e}_{N}^{\sigma}$, given by (36) for $N \in\{0,1,2,3,4,5\}$, in Example 2 .

\begin{tabular}{lllllll}
\hline Error & $N=0$ & $N=1$ & $N=2$ & $N=3$ & $N=4$ & $N=5$ \\
\hline$\hat{e}_{N}^{\mu}$ & 8.72169 & 5.16234 & 1.63597 & 0.322245 & 0.0433663 & 0.00424756 \\
$\hat{e}_{N}^{\sigma}$ & 3.48410 & 3.43451 & 0.566798 & 0.331733 & 0.0367053 & 0.00379717 \\
\hline
\end{tabular}

expressions

$$
\hat{e}_{N}^{\mu}=\int_{0}^{2.75}\left|\mu_{x}^{N+1}(t)-\mu_{x}^{N}(t)\right| \mathrm{d} t, \quad \hat{e}_{N}^{\sigma}=\int_{0}^{2.75}\left|\sigma_{x}^{N+1}(t)-\sigma_{x}^{N}(t)\right| \mathrm{d} t .
$$

\section{Conclusions}

Many important differential equations belonging to the Mathematical Physics and other areas related to Applied Mathematics are formulated through second-order linear differential equations whose coefficients are analytic about ordinary points. Taking into account the physical meaning of these equations, it is natural to consider randomness in their coefficients and initial conditions. In this paper we have computed approximations to the first probability density function of the solution stochastic process of such significant equations under very general hypotheses. The most important issues of our contribution are, firstly, statistical dependence among input random variables has been considered. This approach enables us to deal with the more standard scenario, where input parameters are assumed to be independent random variables, as a particular case. Secondly, uncertainty has been considered in very general point of view since a wide range of probability distributions are allowed as input parameters, including beta, exponential, uniform, gamma, gaussian, etc. This is an important difference against the Itô-type approach, where uncertainty into stochastic differential equations is formulated through the differential of brownian motion or Wiener process, usually referred to as, white noise, which is a gaussian. Thirdly, we point out that an important advantage of computing approximations of the first probability density function of the solution stochastic process allows for computing approximations of the mean and the variance just as merely particular cases. Therefore, our contribution addresses a quite general problem. Finally, we would like to notice that we plan to extend our analysis to second-order linear differential equations with random analytic coefficients about regular singular points in the future work.

\section{Conflict of interest statement}

The authors declare that there is no conflict of interests regarding the publication of this article.

\section{Acknowledgments}

This work has been partially supported by the Ministerio de Economía y Competitividad grant MTM2013-41765-P. Ana Navarro Quiles acknowledges the doctorate scholarship granted by Programa de Ayudas de Investigación y Desarrollo (PAID), Universitat Politècnica de València.

\section{References}

[1] G. Calbo, J.C. Cortés, L. Jódar, Random Hermite differential equations: mean square power series solutions and statistical properties, Appl. Math. Comput. 218 (7) (2011) 3654-3666, doi:10.1016/j.amc.2011.09.008. 
[2] J.C. Cortés, L. Jódar, J. Camacho, L. Villafuerte, Random Airy type differential equations: Mean square exact and numerical solutions, Comput. Math. Appl. 60 (5) (2010) 1237-1244, doi:10.1016/j.camwa.2010.05.046.

[3] A.K. Golmankhaneh, N.A. Porghoveh, D. Baleanu, Mean square solutions of second-order random differential equations by using Homotopy analysis method, Romanian Rep. Phys. 65 (2013) 350-362.

[4] L. Villafuerte, C.A. Braumann, J.C. Cortés, L. Jódar, Random differential operational calculus: theory and applications, Comput. Math. Appl. 59 (1) (2010) 115-125, doi:10.1016/j.camwa.2009.08.061.

[5] T.T. Soong, Random Differential Equations in Science and Engineering, Academic Press, New York, 1973.

[6] A.R. Khudair, S.A.M. Haddad, S. Khalaf, Mean square solutions of second-order random differential equations by using the differential transformation method, Open J. Appl. Sci. 6 (2016) 287-297, doi:10.4236/ojapps.2016.64028.

[7] L. Villafuerte, B.M. Chen-Charpentier, A random differential transform method: theory and applications, Appl. Math. Lett. 25 (2012) 1490-1494, doi:10. 1016/j.aml.2011.12.033.

[8] M.A. El-Tawil, The approximate solutions of some stochastic differential equations using transformations, Appl. Math. Comput. 164 (1) (2005) 167-178, doi:10.1016/j.amc.2004.04.062.

[9] B. Kegan, R.W. West, Modeling the simple epidemic with deterministic differential equations and random initial conditions, Math. Biosci. 195 (5) (2005) 179-193, doi:10.1016/j.mbs.2005.02.004.

[10] M.A. El-Tawil, W. El-Tahan, A. Hussein, Using FEM-RVT technique for solving a randomly excited ordinary differential equation with a random operator, Appl. Math. Comput. 187 (2) (2007) 856-867, doi:10.16/j.amc.2006.08.164.

[11] M.C.C. Cunha, F.A. Dorini, Statistical moments of the solution of the random Burgers-Riemann problem, Math. Comput. Simul. 79 (2014) 1440-1451, doi:10.1016/j.matcom.2008.06.001.

[12] L.T. Santos, F.A. Dorini, M.C.C. Cunha, The probability density function to the random linear transport equation, Appl. Math. Comput. 216 (5) (2010) 1524-1530, doi:10.16/j.amc.2010.03.001.

[13] A. Hussein, M.M. Selim, Solution of the stochastic transport equation of neutral particles with anisotropic scattering using RVT technique, Appl. Math. Comput. 213 (1) (2009a) 250-261, doi:10.1016/j.amc.2009.03.016.

[14] A. Hussein, M.M. Selim, A developed solution of the stochastic Milne problem using probabilistic transformations, Appl. Math. Comput. 216 (10) (2009b) 2910-2919, doi:10.1016/j.amc.2010.04.003.

[15] M.A. El-Tawil, M.A. Sohaly, Mean square numerical methods for initial value random differential equations, Open J. Discret. Math. 1 (2011) 66-84, doi:10.4236/ojdm.2011.12009.

[16] A. Hussein, M.M. Selim, Solution of the stochastic radiative transfer equation with Rayleigh scattering using RVT technique, Appl. Math. Comput. 218 (13) (2012) 7193-7203, doi:10.1016/j.amc.2011.12.088.

[17] M.C. Casabán, J.C. Cortés, J.V. Romero, M.D. Roselló, Determining the first probability density function of linear random initial value problems by the random variable transformation (RVT) technique: a comprehensive study, Abstr. Appl. Anal. 2014-ID248512 (2014a) 1-25, doi:10.1155/2013/248512.

[18] M.C. Casabán, J.C. Cortés, J.V. Romero, M.D. Roselló, Probabilistic solution of random homogeneous linear second-order difference equations, Appl. Math. Lett. 34 (2) (2014b) 27-32, doi:10.1016/j.aml.2014.03.0102.

[19] M.C. Casabán, J.C. Cortés, J.V. Romero, M.D. Roselló, Probabilistic solution of random SI-type epidemiological models using the Random Variable Transformation technique, Commun. Nonlinear Sci. Numer. Simul. 24 (1-3) (2015) 86-97, doi:10.1016/j.cnsns.2014.12.016.

[20] F.A. Dorini, M.S. Cecconello, M.B. Dorini, On the logistic equation subject to uncertainties in the environmental carrying capacity and initial population density, Commun. Nonlinear Sci. Numer. Simul. 33 (2016) 160-173, doi:10.1016/j.cnsns.2015.09.009.

[21] G. Teschl, Ordinary Differential Equations and Dynamical Systems, American Mathematical Society, Providence, 2012.

[22] F. Verhulst, Nonlinear Differential Equations and Dynamical Systems, Springer-Verlag, Berlin Heidelberg, 1996.

[23] C. Fernández, F.J. Vázquez, J.M. Vegas, Differential and Difference Equations. Dynamical Systems, Thomson, Madrid, 2003.

[24] H.L. Royden, P.M. Fitzpatrick, Real Analysis, Pearson Prentice Hall, Taiwan, 2010. 